Research Article

\title{
Ultrasonic Total Focusing Imaging Method of Multilayer Composite Structures Using the Root-Mean-Square (RMS) Velocity
}

\author{
Xufei Guo $\left.{ }^{10}\right)^{1,2}$ and Yan Han $\mathbb{D}^{1}$ \\ ${ }^{1}$ Shanxi Key Laboratory of Signal Capturing \& Processing, North University of China, Taiyuan 030051, China \\ ${ }^{2}$ Department of Physics, Luliang University, Luliang 033000, China \\ Correspondence should be addressed to Yan Han; hanyan@nuc.edu.cn
}

Received 13 May 2021; Revised 13 September 2021; Accepted 13 October 2021; Published 29 October 2021

Academic Editor: Antonio Caggiano

Copyright (c) 2021 Xufei Guo and Yan Han. This is an open access article distributed under the Creative Commons Attribution License, which permits unrestricted use, distribution, and reproduction in any medium, provided the original work is properly cited.

\begin{abstract}
Multilayer composite structures have been widely used in industrial manufacturing, and nondestructive testing of these multilayer structures is to ensure their reliable quality and performance. Currently, ultrasonic total focusing method (TFM) imaging using full-matrix capture (FMC) technology has been proven to sense small defects in a single homogeneous medium and improve the imaging signal-to-noise ratio. However, these algorithms cannot be accurately applied to imaging of multilayer composite structures, due to the acoustic impedance variation and because reflection and refraction occur at the interface between the layers, which makes it very difficult to calculate the ultrasonic propagation path and time. To solve this problem, a root-mean-square (RMS) velocity algorithm for total focusing imaging of multilayer structures is proposed in the article. Based on the theory of RMS velocity for processing of seismic data, the approximated delays can be easily and quickly calculated by a hyperbolic time-distance relationship under circumstances of short lateral distance and horizontal layers. The performance of the proposed algorithm is evaluated by total focusing imaging of a two-layer medium with drilled holes and conducted by the finite element simulation. To further improve imaging efficiency, the partial data in the full-matrix data were used for imaging which is the simplified matrix focusing method (SFM). The results verify that the proposed methods are capable of total focusing imaging of two-layered structures. However, the imaging performance and efficiency of these algorithms are different.
\end{abstract}

\section{Introduction}

Multilayer structures play an important role in the production and manufacturing of industrial products, such as solid rocket motors, aircraft skin, and generator blade, due to their advantages in corrosion protection, wear-resistant and strength enhancement, and so on $[1,2]$. In order to ensure the reliable quality and performance of these layered structures, nondestructive testing (NDT) of these structures is essential [3]. Ultrasonic NDT is the well-established method that is widely applied to a broad range of applications, especially the detection of multilayer structure, mainly including the characterization of its material properties, the judgment of interface bonding quality, and the determination of structural consistency.
At present, the ultrasonic phased array is a well-known nondestructive testing (NDT) method, and its application has increased dramatically [4-7]. Compared with singleelement ultrasonic transducers, ultrasonic arrays have advantages in sensitivity, coverage, etc. [8]. Conventionally, the ultrasonic phased array method is based on the same time delay law by controlling each array element to form a coherent beam and receiving echo signals to image [9]. In order to further improve the imaging resolution, different postprocessing imaging algorithms have been developed, such as dynamic focusing [10], compound imaging [11], and full-matrix imaging [12]. In the time domain, full-matrix capture (FMC) excites each array element in turn, and all array elements (including transmitting array elements) receive echo signals. The total focus method (TFM) realizes 
imaging by processing the captured full-matrix data [13]. Nevertheless, the total focus method is time-consuming due to the large amount of processing data [12]. To improve imaging efficiency, different total focus algorithms have been proposed [14-19]. Note that both the algorithms based on TFM are only suitable for homogeneous and isotropic materials. Therefore, these algorithms are invalid for multilayer structures as the acoustic impedance varies between different layers.

Several methods to ultrasonic imaging of multilayer structures were developed. The ray-tracing method $[20,21]$ is used for imaging by accurately calculating the sound beam propagation path and time, but the complexity of iterative operation reduces its imaging efficiency. Ultrasonic imaging with virtual sources $[22,23]$ is introduced to improve the calculating time. However, this approach is susceptible to the detection characteristics and cannot obtain high imaging resolution. Recently, the method in seismic imaging is used for reference, and the phase shift migration (PSM) algorithm [21] based on the plane wave theory was employed to synthetic aperture focusing technique (SAFT) of multilayer structures. To improve calculation efficiency and imaging resolution, several frequency-domain ultrasound imaging algorithms based on PSM are proposed [24-28]. Unfortunately, the programming of this method is both complicated and needed.

In this work, we propose an ultrasonic TFM, based on root-mean-square (RMS) velocity, which is derived from processing of seismic data. Using the RMS velocity, the ultrasonic TFM in single medium can be modified for applied on multilayer structures. The remainder of this work is organized as follows. In Section 2, the TFM of single medium, traditional multilayer structure TFM, the RMS velocity theory, and the proposed multilayer TFM are presented. Simulation has been conducted in to evaluate imaging performance of multilayer TFM, and the results are presented in Section 3. Finally, the main conclusions of the paper are in Section 4.

\section{Theory and Methods}

2.1. TFM in a Single Medium. Consider the typical FMC setup shown in Figure 1. Each transducer is excited in turn along a line in the $x$ direction, and all transducers (including transmitting transducer) receive echo signals from a scatterer. Finally, a full-matrix data is obtained. The TFM is defined by

$$
I_{p}\left(x_{p}, z_{p}\right)=\sum_{i=1}^{N} \sum_{j=1}^{N} A_{i j}\left(t_{i j}\left(x_{p}, z_{p}\right)\right),
$$

where $\left(x_{p}, z_{p}\right)$ is coordinates of scatterer $P, A_{i j}$ is a amplitude intensity of scatterer $P$ excited by transducer $i$ and received by transducer $j, t_{i j}\left(x_{p}, z_{p}\right)$ is the time of flight staring from the transducer $i$, passing through to the scatterer $P$ and arriving at the transducer $j$, and $N$ is the number of transducers. The time of flight is calculated by

$$
t_{i j}\left(x_{p}, z_{p}\right)=\frac{\sqrt{\left(x_{i}-x_{p}\right)^{2}+z_{p}^{2}}+\sqrt{\left(x_{j}-x_{p}\right)^{2}+z_{p}^{2}}}{c},
$$

where $c$ is ultrasonic velocity in the single medium and $\left(x_{i}, 0\right)$ and $\left(x_{j}, 0\right)$ are the Cartesian coordinates of the transmitting transducer and the receiving transducer, respectively. Assume that $t_{0}\left(z_{p}\right)=z_{p} / c$ is the normal incidence one-way flight time from $i^{\text {th }}$ ultrasonic transducer to the scatterer $P$ in the single medium. The time of flight can be modified as

$$
t_{i j}\left(x_{p}, z_{p}\right)=\sqrt{t_{0}^{2}\left(z_{p}\right)+\frac{\left(x_{i}-x_{p}\right)^{2}}{c^{2}}}+\sqrt{t_{0}^{2}\left(z_{p}\right)+\frac{\left(x_{j}-x_{p}\right)^{2}}{c^{2}}} .
$$

Consider the energy of weight of different transducers to the imaging area, and Equation (1) can be modified by

$$
I_{p}\left(x_{p}, z_{p}\right)=\sum_{i=1}^{N} \sum_{j=K}^{M} A_{i j}\left(t_{i j}\left(x_{p}, z_{p}\right)\right), \quad 1<K, M<N .
$$

Since the amount of postprocessing data is part of the full-matrix data, theoretically, the calculation time of this simplified matrix focusing method (SFM) will also be reduced. To superimpose and offset the random noise of positive and negative values, it is necessary to normalize the captured full-matrix data $[18,19]$. The amplitude is normalized by

$$
\left\{\begin{array}{l}
I_{p}\left(x_{p}, z_{p}\right)=\frac{I_{p}\left(x_{p}, z_{p}\right)}{I_{\max }\left(x_{p}, z_{p}\right)} \times 128, \quad I_{p}\left(x_{p}, z_{p}\right) \geq 0, \\
I_{p}\left(x_{p}, z_{p}\right)=-\frac{I_{p}\left(x_{p}, z_{p}\right)}{I_{\min }\left(x_{p}, z_{p}\right)} \times 127, \quad I_{p}\left(x_{p}, z_{p}\right)<0 .
\end{array}\right.
$$

\subsection{Traditional Multilayer Structure TFM (TML-TFM).} The principle of traditional multilayer structure TFM [29] is shown in Figure 2. The height of layer 1 is $h$. The propagation speed of ultrasonic waves in layer 1 and layer 2 are $c 1$ and $c 2$, respectively. Assuming that the incident point of the $i$ th element at the interface of the double-layer structure is $S_{1}$, the incident angle and refraction angle are $\theta_{1}$ and $\theta_{2}$, respectively, and $P$ represents any point in the imaging area.

In Figure 2, the following equation can be obtained by

$$
x_{p}=h \tan \theta_{1}+\left(z_{p}-h\right) \tan \theta_{2} .
$$

According to Snell's law, the following equation can be obtained by

$$
\frac{\sin \theta_{1}}{\sin \theta_{2}}=\frac{c_{1}}{c_{2}} .
$$




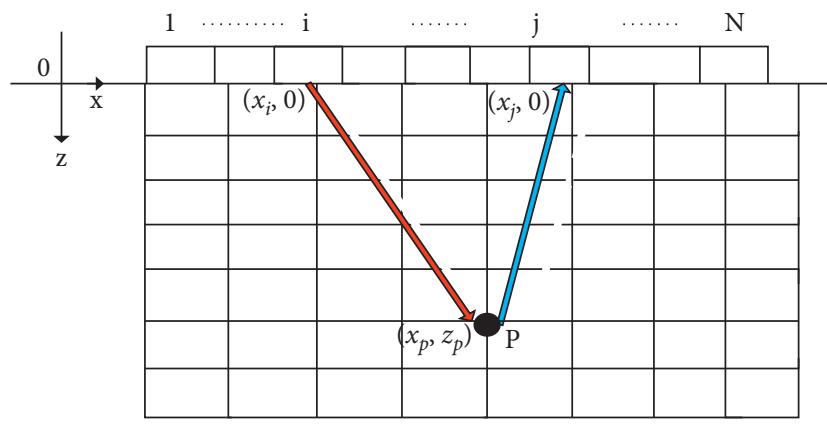

FIgURE 1: Schematic diagram of FMC and TFM.

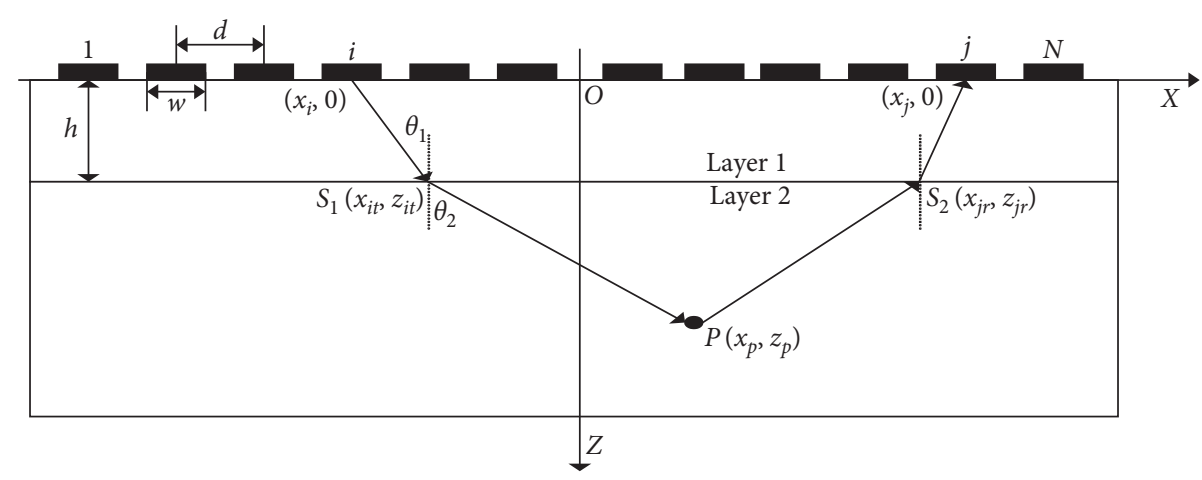

FIgURE 2: The principle of traditional multilayer structure TFM.

Then, the flight time of the ultrasonic wave reflected from the $i$ th element through point $\mathrm{P}$ to the $j$ th element in the double-layer structure can be expressed as

$$
\begin{aligned}
t_{i j}\left(x_{p}, z_{p}\right)= & \frac{\sqrt{\left(x_{i}-x_{i t}\right)^{2}+z_{i t}^{2}}+\sqrt{\left(x_{j}-x_{j r}\right)^{2}+z_{i t}^{2}}}{c_{1}} \\
& +\frac{\sqrt{\left(x_{p}-x_{i t}\right)^{2}+\left(z_{p}-z_{i t}\right)^{2}}+\sqrt{\left(x_{p}-x_{j r}\right)^{2}+\left(z_{p}-z_{j r}\right)^{2}}}{c_{2}} .
\end{aligned}
$$

In the double-layer structure, because the acoustic impedances of the two materials are different, the ultrasonic waves are reflected and refracted at the interface. According to Fermat's principle, the speed of sound always travels along the path with the shortest flight time. Therefore, the minimum value of $t_{i j}\left(x_{p}, z_{p}\right)$ needs to be solved as the time index value of the full-matrix data processing.

2.3. Multilayer TFM Based on the RMS Velocity (RML-TFM). In the case of multilayer structures, the ultrasonic propagation path becomes a bit more complicated. The ultrasonic propagation path is illustrated in Figure 3. Because these layers have different sound velocities, reflection and refraction occur at the interfaces. In this case, the precise time of flight and delay between the scatterer and the each transducer cannot be calculated analytically [20]. In seismic signal processing technology, the RMS velocity is often adopted to calculate the migration imaging velocity in the geological layers of the Earth [30-32]. And, some seismic signal processing methods [33] are also suitable for ultrasonic data processing. Therefore, the RMS velocity model derived from seismic data processing can approximately calculate the time delay of ultrasonic propagation in multilayer structures.

In Figure 3, it is assumed that the ultrasound array transducer is placed on top of layer 1 , and the layers are, respectively, named $1,2, \ldots, L$. The normal incidence oneway flight time from $i^{\text {th }}$ ultrasonic transducer to the scatterer $P$ in layer number $L$ is calculated by

$$
t_{0}\left(z_{p}\right)=\left(\sum_{k=1}^{L-1} \frac{h_{k}}{c_{k}}\right)+\frac{\Delta z}{c_{L}}
$$




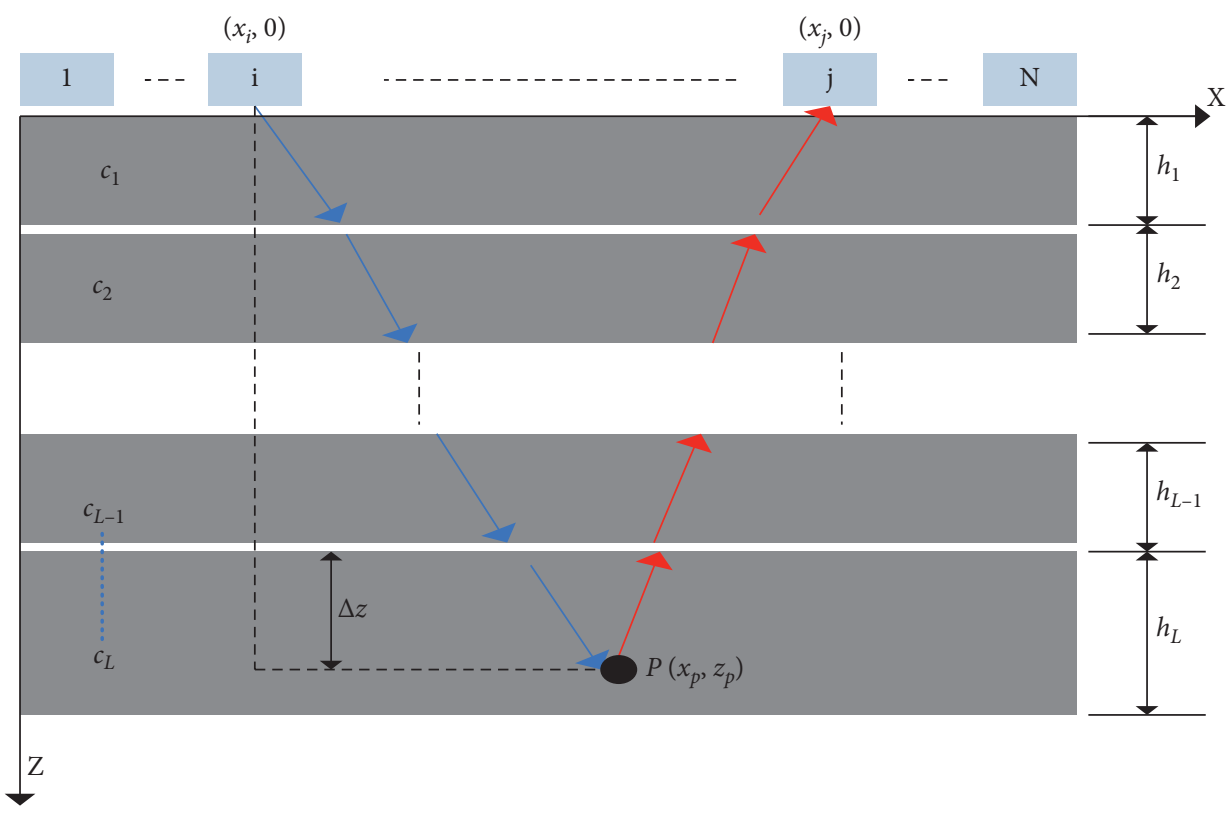

FIGURE 3: Wave propagation through multilayer with different sound velocities.

where $h_{k}$ and $c_{k}$ are the thickness and wave velocity of layer $k$, respectively, and $\Delta z=z_{p}-\sum_{k=1}^{L-1} h_{k}$ is vertical distance from the scatterer $P$ to the interface between the last two layers. The RMS velocity of multilayer structures is defined by

$$
c_{\mathrm{rms}}\left(z_{p}\right)=\sqrt{\frac{\left(\sum_{k=1}^{L-1} c_{k}^{2} t_{k}\right)+c_{L}^{2}\left(t_{0}\left(z_{p}\right)-\sum_{k=1}^{L-1} t_{k}\right)}{t_{0}\left(z_{p}\right)}},
$$

where $t_{k}=h_{k} / c_{k}$ is the normal incidence one-way flight time of layer $k$. Substituting equation (9), $\Delta z=z_{p}-\sum_{k=1}^{L-1} h_{k}$, and $h_{k}=c_{k} t_{k}$ into equation (10), the RMS velocity can be modified as

$$
c_{\mathrm{rms}}\left(z_{p}\right)=\sqrt{\frac{\left(\sum_{k=1}^{L-1} c_{k} h_{k}\right)+c_{L} \Delta z}{t_{0}\left(z_{p}\right)}} .
$$

Based on the law of RMS velocity, it is possible that the time of flight starting from the transducer $i$, passing through to the scatterer $P$, and arriving at the transducer $j$ is derived.

In multilayer structures, the flight time in a multilayer structure is similar to that in the single medium case:

$$
\begin{aligned}
t\left(z_{p}, x_{p}, x_{i}, x_{j}\right)= & \sqrt{t_{0}^{2}\left(z_{p}\right)+\frac{\left(x_{i}-x_{p}\right)^{2}}{c_{\mathrm{rms}}^{2}\left(z_{p}\right)}} \\
& +\sqrt{t_{0}^{2}\left(z_{p}\right)+\frac{\left(x_{j}-x_{p}\right)^{2}}{c_{\mathrm{rms}}^{2}\left(z_{p}\right)}} .
\end{aligned}
$$

The conclusion can be obtained from [30] that expression (equation (12)) for $t$ is equivalent to ignoring the higher-order part of the Taylor series expansion of $\left(x_{p}-\right.$ $\left.x_{i}\right)=0$ for $t$. Therefore, this corresponding flight time is an approximate analytical solution. Using the imaging equation that was applied for TFM in a single medium (equation (1)), the modified equation (equation (12)) for $t$ can be adopted for full focusing in multilayer structures. We call this algorithm multilayer TFM (RML-TFM). If the expression (equation (4)) is combined with the expression (equation (12)), this method can also be used for focusing imaging of multilayer structures. This algorithm was referred as multilayer SFM (RML-SFM).

\section{Experiments and Results}

3.1. Experimental Conditions. To further verify the performance of our proposed imaging method, ultrasonic TFM of three two-layer structures are conducted in finite element method (FEM) simulation. These three two-layer structures are composed of acrylic material and aluminum. For clarity, their geometry and the corresponding defects are depicted in Figure 4 . These three multilayer structures are numbered model 1, model 2, and model 3, respectively. A 32-element linear phased array is configured for imaging simulation, and the array specifications are shown in Table 1 . In the simulation process, only the ultrasonic longitudinal wave generated by the array is considered, and the shear wave is ignored. In acrylic and aluminum materials, the ultrasonic longitudinal wave propagation velocity approximately is $2720 \mathrm{~m} / \mathrm{s}$ and $6260 \mathrm{~m} / \mathrm{s}$, respectively.

In this simulation, the finite element software ABAQUS (Dassault, Velizy-Villacoublay, France) was used to generate the full-matrix data. According to the principle of FMC, each array element is separately excited by a sinusoidal signal modulated by the Hanning window, and all array elements receive echo signals. As a result, the full-matrix data of $32 \times$ 32 were captured with a sampling frequency of $50 \mathrm{MHz}$ and imported into MATLAB R2016a (Math-Works, Natick, MA, 


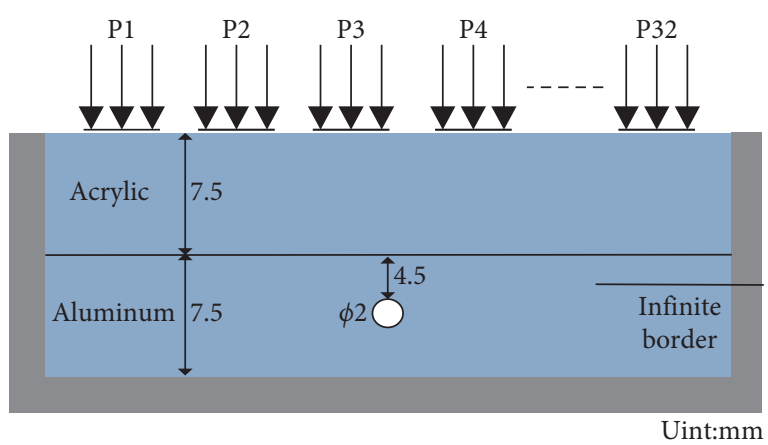

(a)

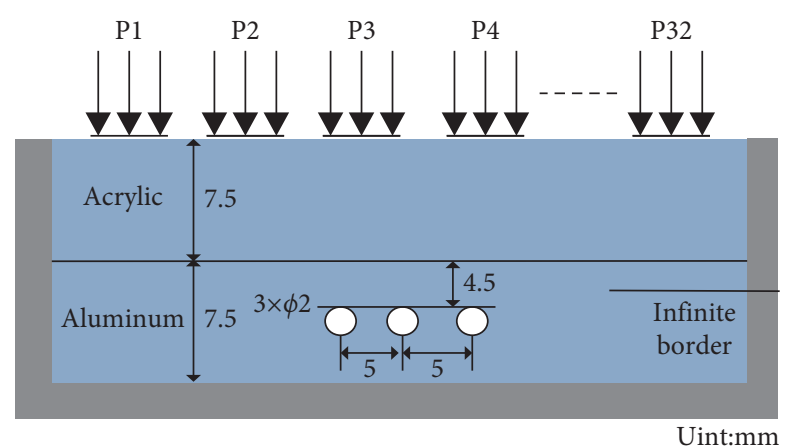

(b)

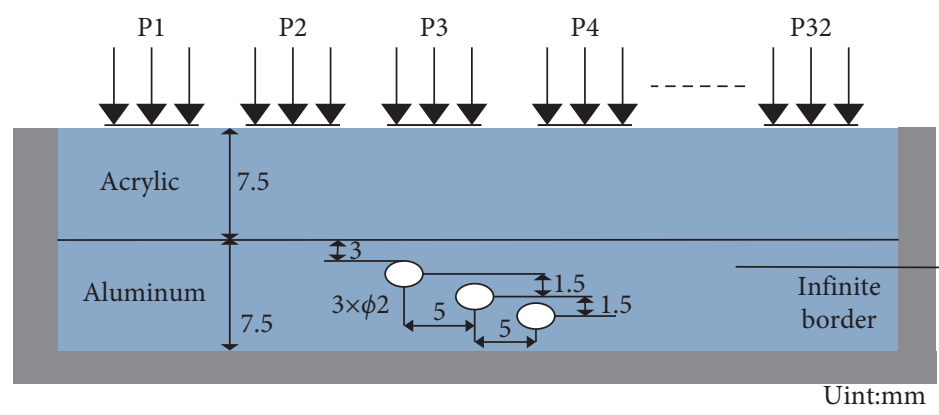

(c)

Figure 4: The geometry of two-layer structure. (a) Model 1. (b) Model 2. (c) Model 3.

TABLE 1: The specifications of the ultrasonic phased array.

\begin{tabular}{lr}
\hline Parameter & Value \\
\hline Number of elements & 32 \\
Element width & $0.4 \mathrm{~mm}$ \\
Element pitch & $0.1 \mathrm{~mm}$ \\
Sampling time interval & $2 e-8 \mathrm{~s}$ \\
Center frequency & $5 \mathrm{MHz}$ \\
\hline
\end{tabular}

USA) for postprocessing with different imaging algorithms. In this paper, the full matrix data are postprocessed by TMLTFM, RML-TFM, and RML-SFM.

\subsection{Results and Discussion}

3.2.1. Imaging Results of Single Medium. This paper uses the experimental setup described in [19] to focus imaging on a single-layer medium, and the imaging results are shown in Figure 5. It can be seen that the imaging quality of the simplified matrix is the same as that of the full matrix. Compared with TFM, the imaging efficiency by using SFM is greatly improved.

3.2.2. Imaging Results of Multilayer Composite Structure. Figure 6 presents the amplitude data for full matrix of model 2. It can be observed from Figure 6 that the fullmatrix data has symmetry. In other words, the upper or lower triangle data can also be used for postprocessing focused imaging. In this article, the full-matrix imaging and simplified-matrix imaging are conducted on these three multilayer structures with different defects. The imaging results of different algorithms are shown in Figures 7-9.

In order to prevent aliasing in space and time, according to the spatial sampling theorem [28], the imaging pixel density in the $x$ and $z$ directions are both $0.1 \mathrm{~mm}$. In Figures 7-9, using traditional methods, there is obvious noise in the near-field area of the ultrasound image. However, using ML-TFM, ML-SFM-1, and ML-SFM-2, it can be observed that there is a pseudointerface at the interface of the two media. Compared with the total focus image based on the root-mean-square velocity, the false defects around every defect in the traditional total focus image are more prominent. Moreover, using ML-SFM-2, these defects display brightness is poor.

The above imaging results show that the imaging quality of the full matrix of a single medium is consistent with the imaging quality of the simplified matrix. However, the imaging quality of the full matrix of the double-layer composite structure based on the root-mean-square velocity is higher than that of the simplified matrix. And, in the fullmatrix image of the double-layer structure, the integrity of the defects is poor. 


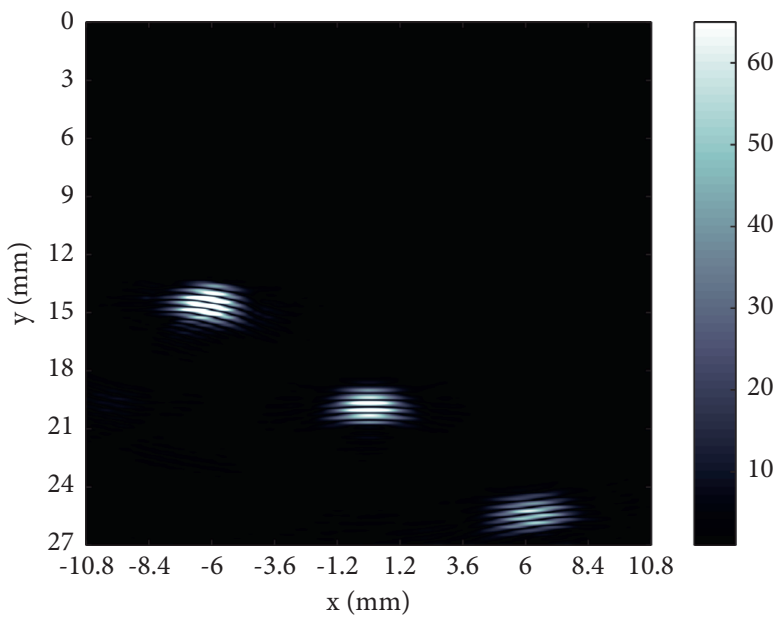

(a)

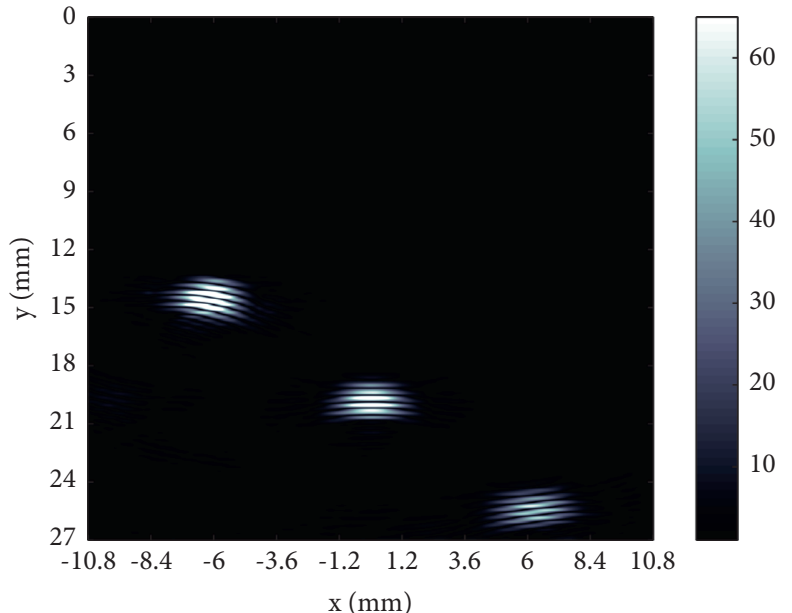

(b)

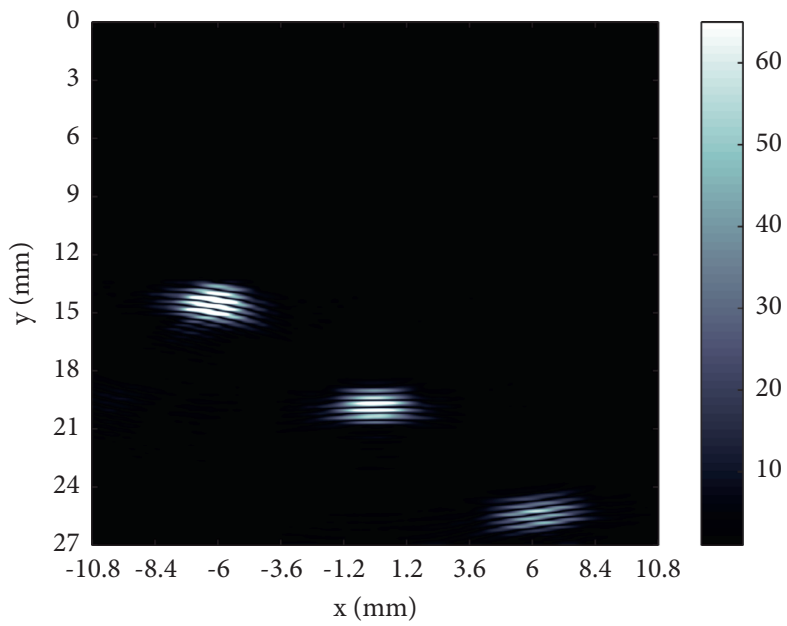

(c)

Figure 5: Focused imaging of a single medium. (a) TFM. (b) SFM-1 (in equation (4), $j=7: 23$ ). (c) SFM-2 (in equation (4), $j=1: i$ ).

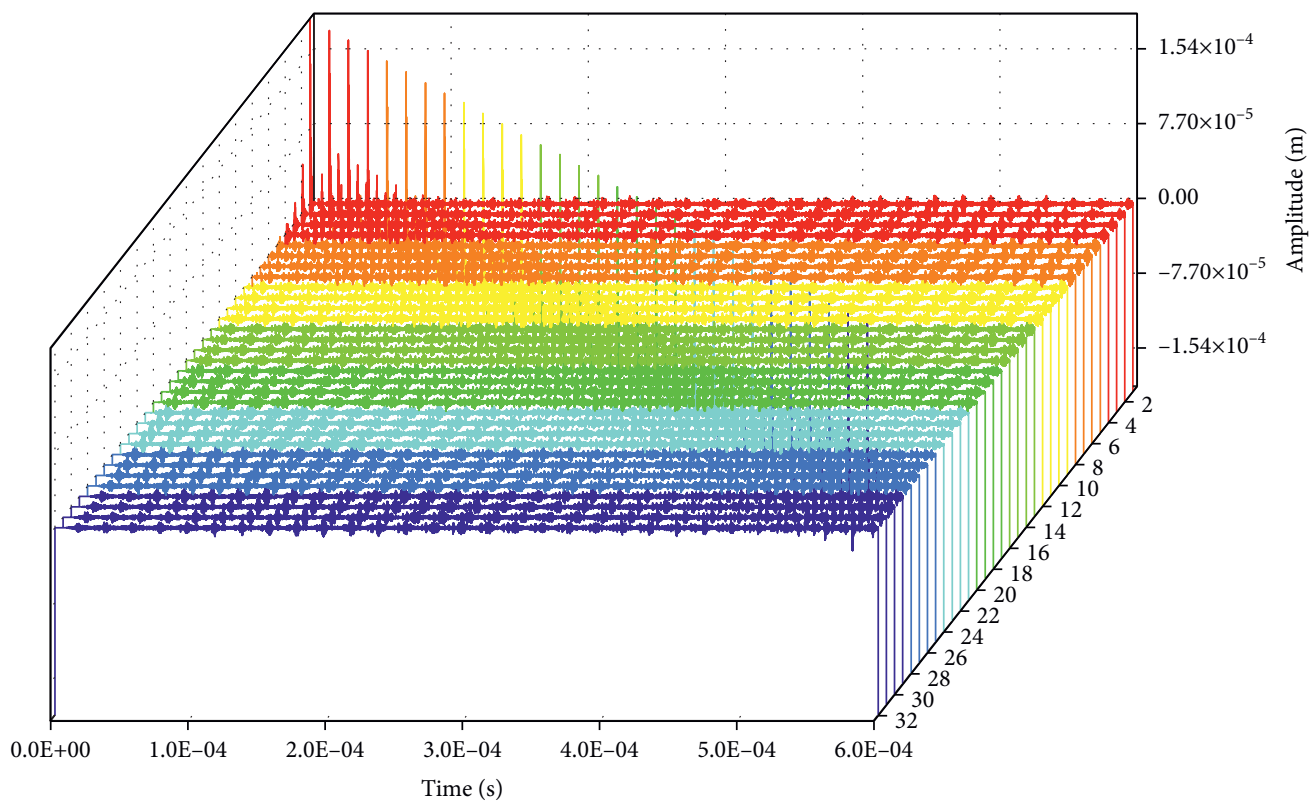

FIgURE 6: The captured full-matrix data of model 2. 


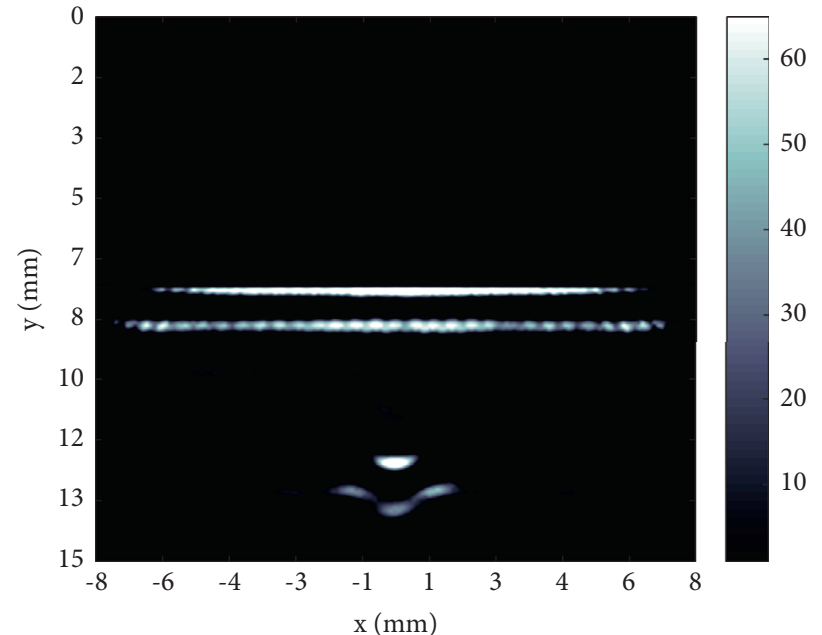

(a)

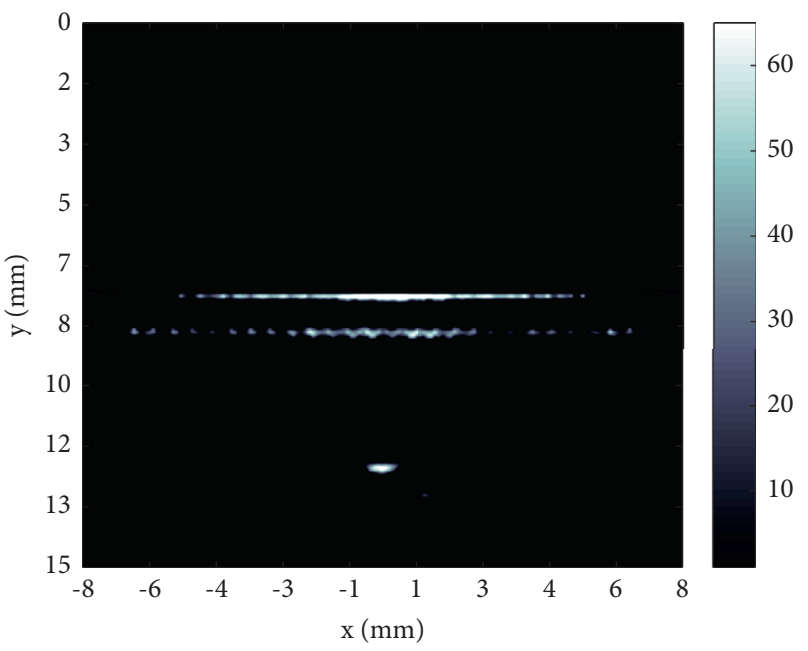

(c)

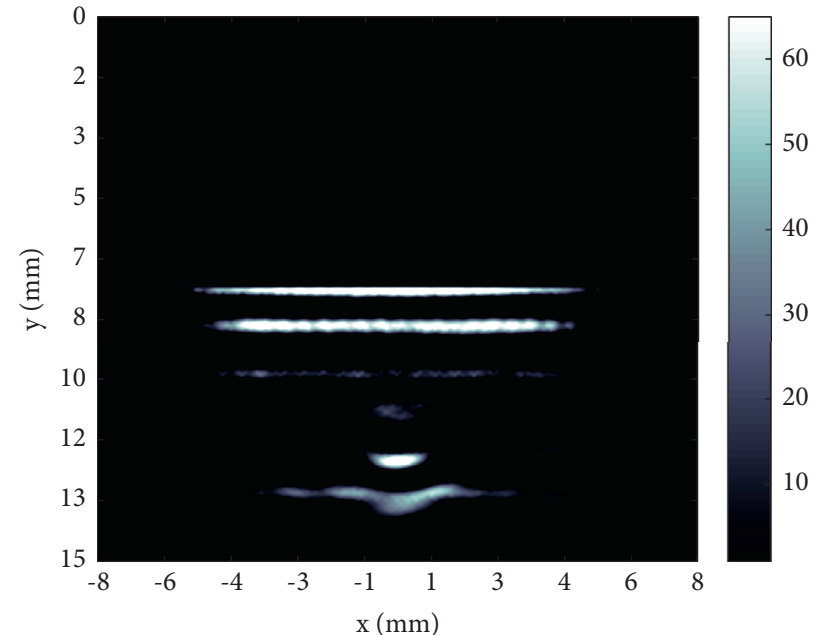

(b)

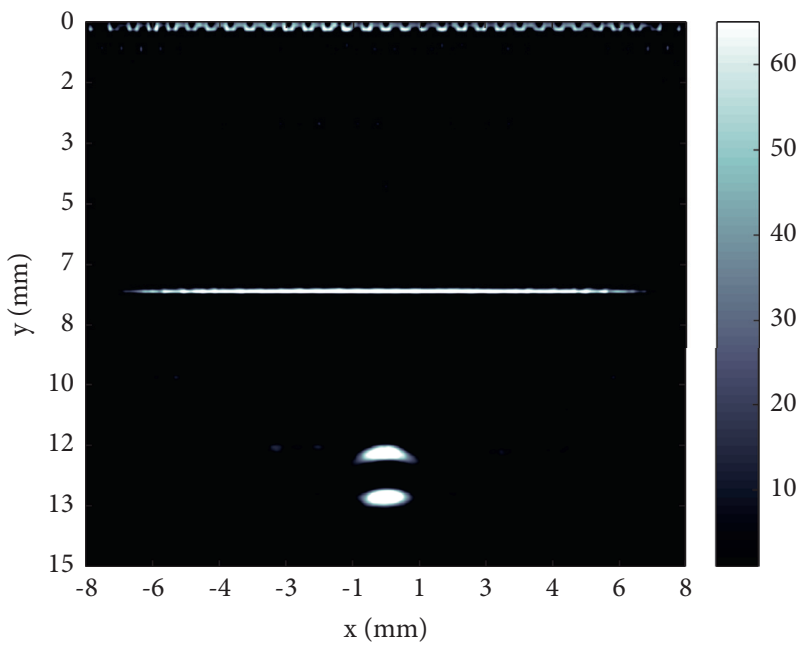

(d)

Figure 7: Focused imaging of model 1: (a) ML-TFM; (b) ML-SFM-1 (in equation (4), $j=7: 23$ ); (c) ML-SFM-2 (in equation (4), $j=1: i$ ); (d) TML-TFM.

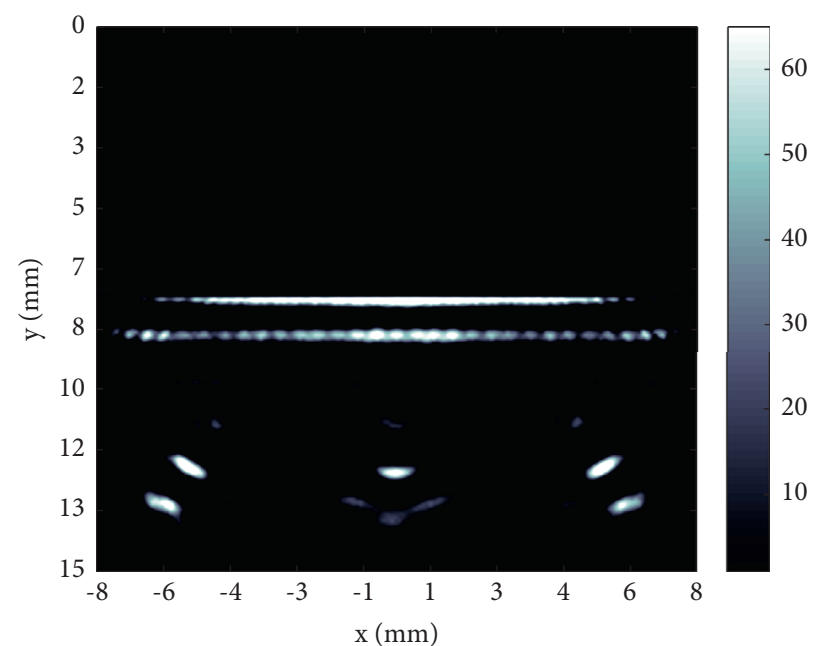

(a)

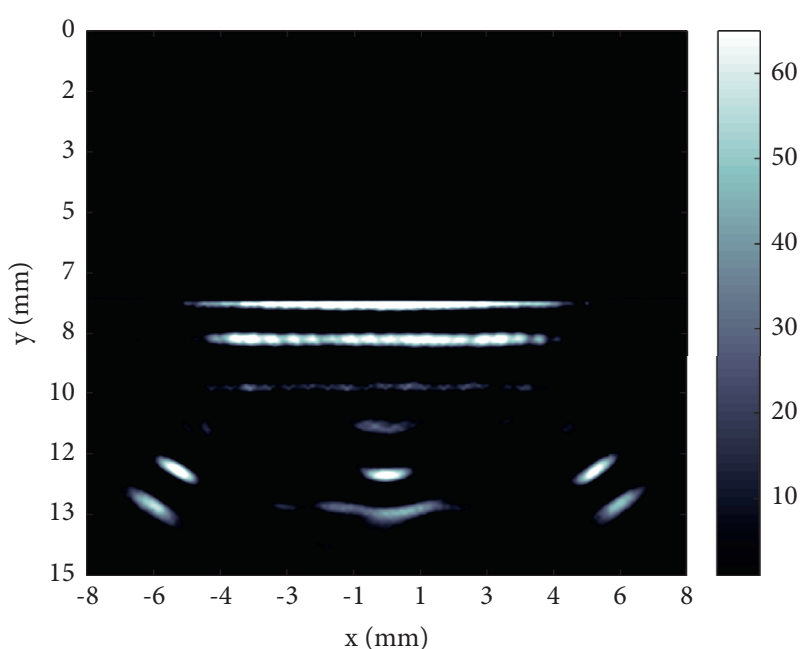

(b)

Figure 8: Continued. 


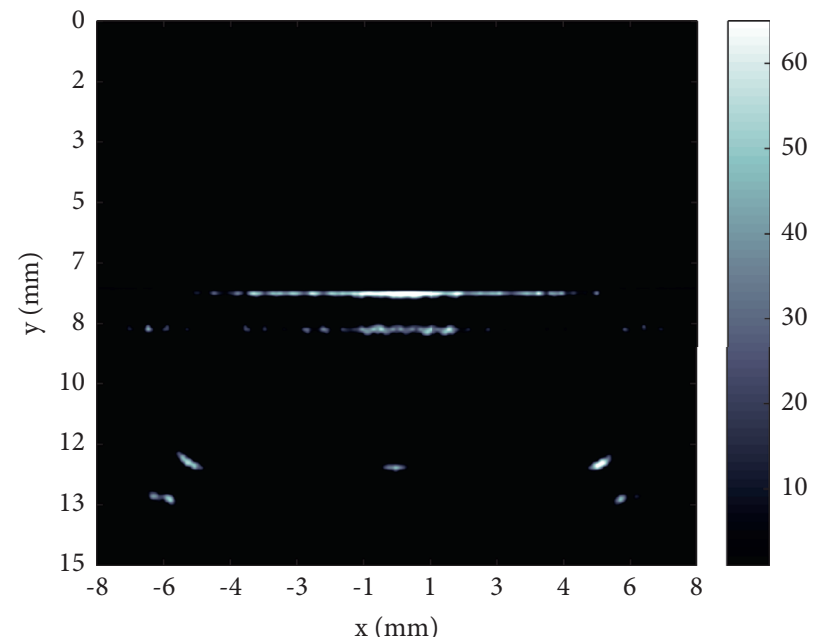

(c)

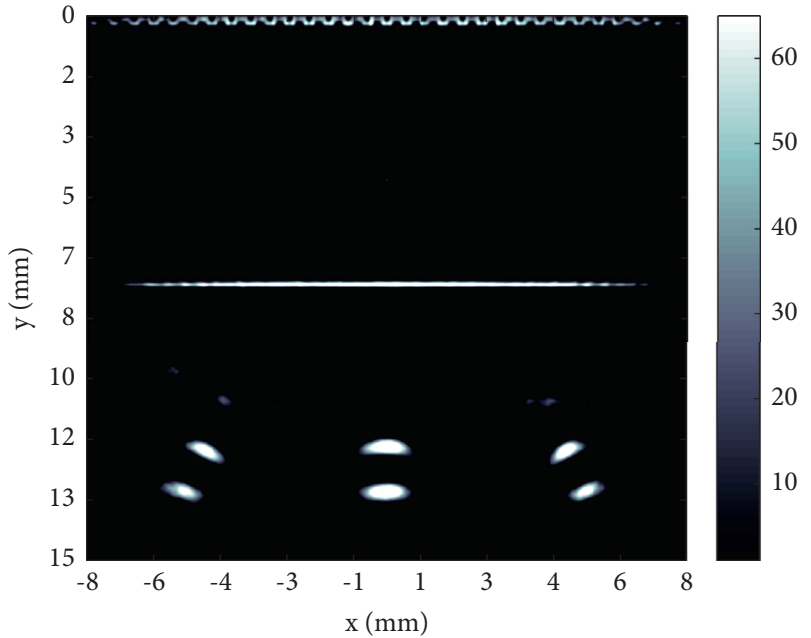

(d)

Figure 8: Focused imaging of model 2: (a) ML-TFM; (b) ML-SFM-1 (in equation (4), $j=7: 23$ ); (c) ML-SFM-2 (in equation (4), $j=1: i$ ); (d) TML-TFM.

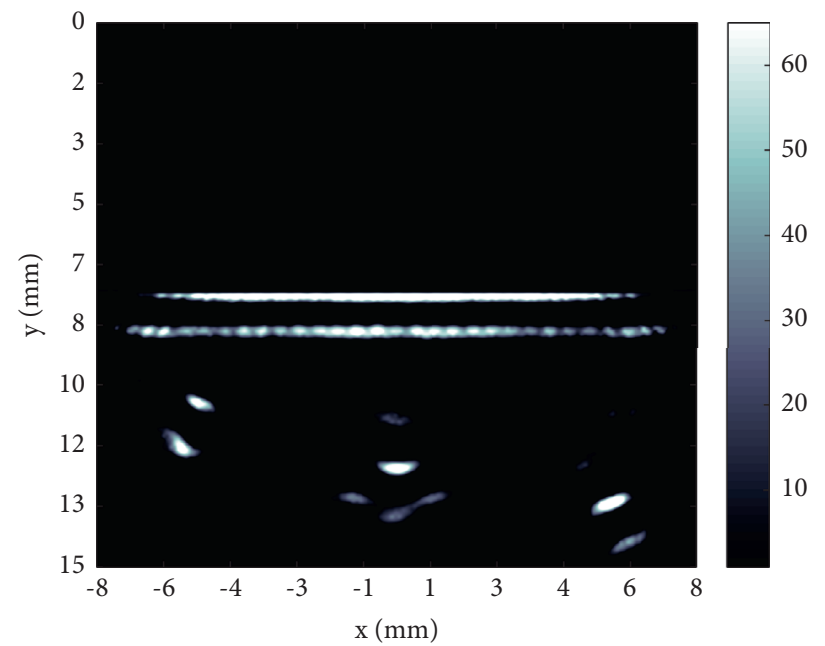

(a)

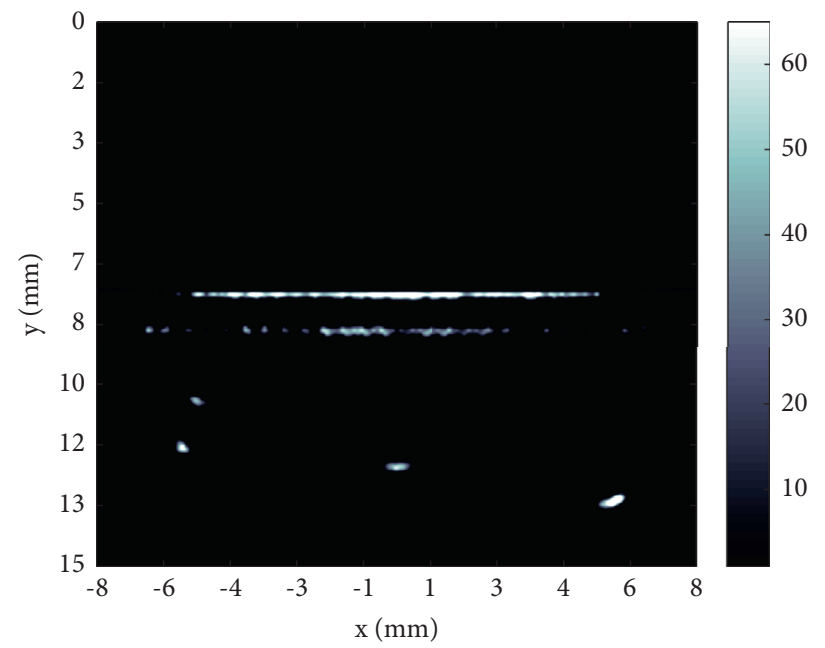

(c)

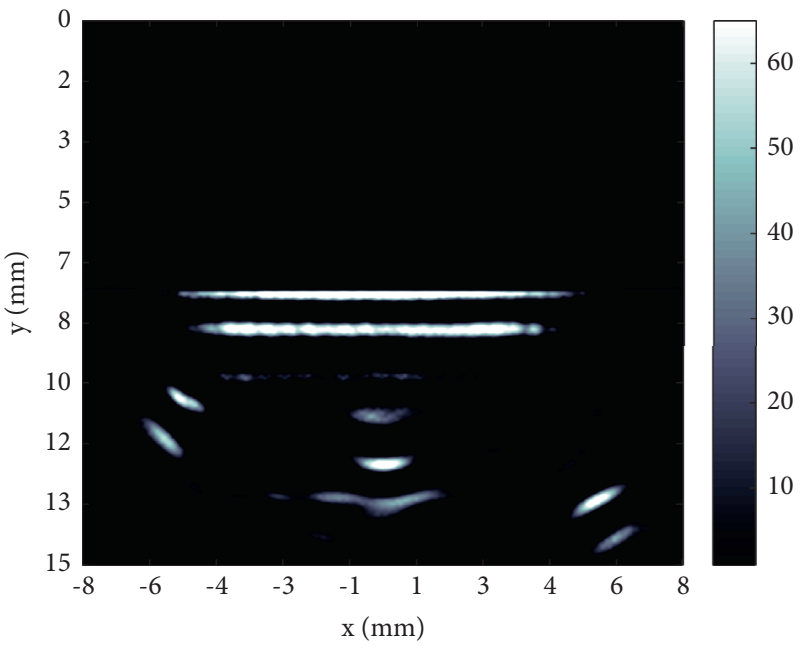

(b)

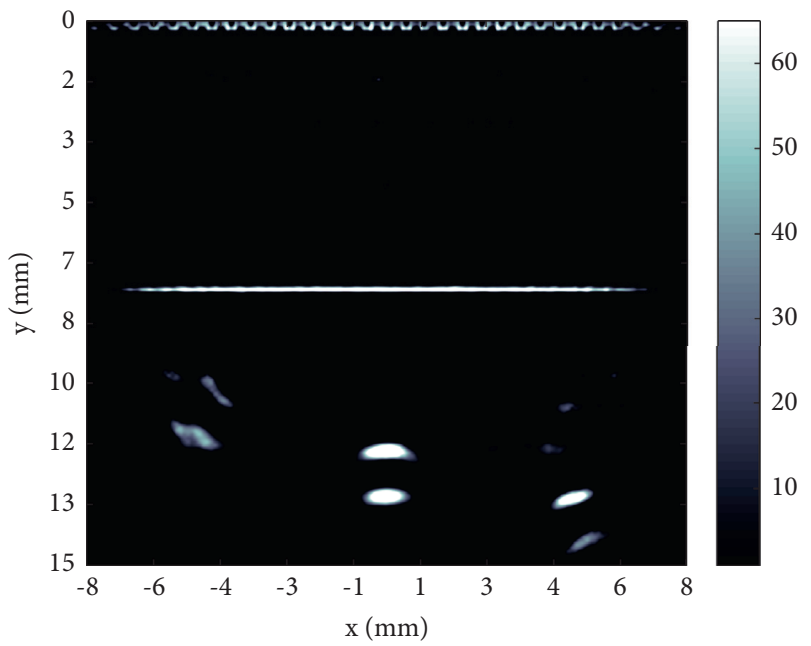

(d)

Figure 9: Focused imaging of model 3: (a) ML-TFM; (b) ML-SFM-1 (in equation (4), $j=7: 23$ ); (c) ML-SFM-2 (in equation (4), $j=1: i$ ); (d) TML-TFM. 


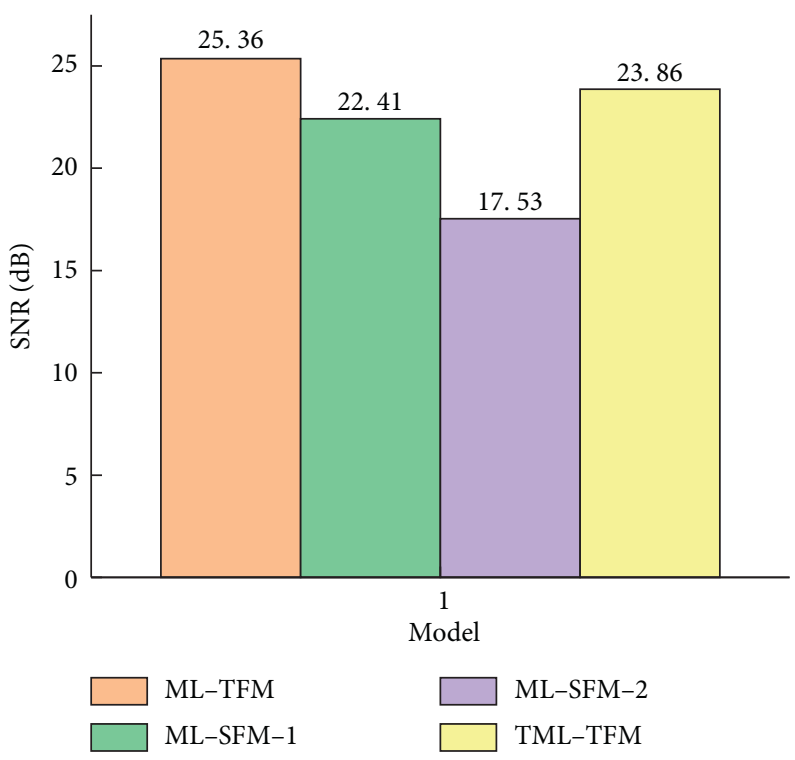

FIGURE 10: SNR of different algorithms.

TABLE 2: Platform configuration.

\begin{tabular}{lr}
\hline Specifications & Related configuration \\
\hline Operating systems & Windows \\
CPU/GHz & Inter Core I5 9300H, 2.4 \\
Ram/GB & 8 \\
GPU & NVIDIA GeForce GTX1650 \\
\hline
\end{tabular}

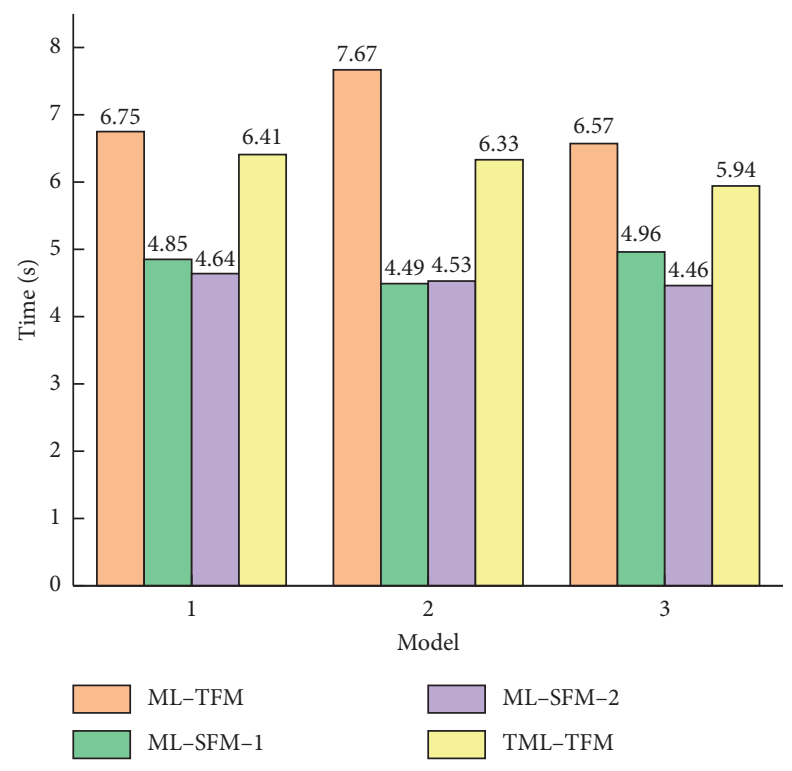

FIgURE 11: Imaging time of different algorithms.

3.2.3. Imaging Signal-to-Noise Ratio (SNR). To quantitatively analyze the performance of different imaging methods, the image signal-to-noise ratio is used to express the relationship between the defect signal and the noise. The signalto-noise ratio for a defect can be expressed $[18,19]$ by

$$
\mathrm{SNR}=20 \times \log _{10}\left(\frac{V_{p-p}}{V_{\text {ave }}}\right),
$$

where $V_{p-p}$ is the peak-to-peak value of the defect signal in the surrounding region and $V_{\text {ave }}$ is the average of noise 
signal. Except for the defects, the rest of the image is regarded as noise signal.

In this paper, model 1 is used as the research object to calculate the defect signal-to-noise ratio of different imaging methods. In Model 1, the defect signal-to-noise ratios of the four different imaging methods are shown in Figure 10. It can be observed that ML-TFM has the best signal-to-noise ratio. And, the SNR of ML-SFM-1 and ML-SFM-2 are relatively low. Moreover, the SNR of TML-TFM is between ML-TFM and ML-SFM.

3.2.4. Imaging Efficiency. The entire experimental computing platform configuration in this article is presented in Table 2. Figure 11 shows the imaging time of these three models using different imaging algorithms, respectively. It can be observed that ML-TFM has the lowest imaging efficiency.

And, the imaging time of ML-SFM-1 and ML-SFM-2 are relatively close. Moreover, the imaging time of TML-TFM is between ML-TFM and ML-SFM.

\section{Conclusions}

In this paper, a multilayer structures ultrasonic full-matrix imaging method based on root-mean-square (RMS) velocity is proposed. The experimental results show that full-matrix imaging based on RMS velocity has the best signal-to-noise ratio and the lowest imaging efficiency. However, the simplified matrix imaging based on RMS velocity has the worst imaging performance and the highest imaging efficiency. The imaging performance and efficiency of the traditional full-matrix imaging method are between the above two. For the multilayer structure, how to maintain high-performance imaging while improving imaging efficiency needs to be studied further.

\section{Data Availability}

The data used to support the findings of this study are available from the corresponding author upon request.

\section{Conflicts of Interest}

The authors declare no conflicts of interest.

\section{Acknowledgments}

This work was supported by the Shanxi Key Laboratory of Signal Capturing and Processing, North University of China (Grant no. ISPT2020-8), and Natural Science Foundation of Shanxi, China (Grant nos. 201801D121156 and 201901D111165).

\section{References}

[1] G.-M. Zhang and D. M. Harvey, "Contemporary ultrasonic signal processing approaches for nondestructive evaluation of multilayered structures," Nondestructive Testing and Evaluation, vol. 27, no. 1, pp. 1-27, 2012.
[2] J. Chen, X. Bai, K. Yang, and B.-F. Ju, "The computations of reflection coefficients of multilayer structure based on the reformulation of Thomson-Haskell method," Ultrasonics, vol. 52, no. 8, pp. 1019-1023, 2012.

[3] C. Li, D. Pain, P. D. Wilcox, and B. W. Drinkwater, "Imaging composite material using ultrasonic arrays," NDT \& E International, vol. 53, pp. 8-17, 2013.

[4] B. W. Drinkwater and P. D. Wilcox, "Ultrasonic arrays for non-destructive evaluation: a review," NDT \& E International, vol. 39, no. 7, pp. 525-541, 2006.

[5] H. J. Hu, X. Zhu, C. H. Wang et al., "Stretchable ultrasonic transducer array three dimensional imaging on complex surfaces," Science Advances, vol. 4, pp. 1-11, 2018.

[6] C. Yuan, C. Xie, L. Li, F. Zhang, and S. M. Gubanski, "Ultrasonic phased array detection of internal defects in composite insulators," IEEE Transactions on Dielectrics and Electrical Insulation, vol. 23, no. 1, pp. 525-531, 2016.

[7] G. D. Connolly, M. J. S. Lowe, J. A. G. Temple, and S. I. Rokhlin, "Correction of ultrasonic array images to improve reflector sizing and location in inhomogeneous materials using a ray-tracing model," Journal of the Acoustical Society of America, vol. 127, no. 5, pp. 2802-2812, 2010.

[8] X. X. Zhao, T. Gang, and B. X. Zhang, "Prediction of radiation beam fields from an array transducer with non-paraxial multiGaussian beam model," Shengxue Xuebao/Acta Acustica, vol. 33, pp. 475-480, 2008.

[9] H. Seo, D.-K. Pyun, and K.-Y. Jhang, "Synthetic aperture imaging of contact acoustic nonlinearity to visualize the closing interfaces using tone-burst ultrasonic waves," $M e$ chanical Systems and Signal Processing, vol. 125, pp. 257-274, 2019.

[10] M. Flesch, M. Pernot, J. Provost et al., "4Din vivoultrafast ultrasound imaging using a row-column addressed matrix and coherently-compounded orthogonal plane waves," Physics in Medicine and Biology, vol. 62, no. 11, pp. 4571-4588, 2017.

[11] D. Garcia, L. L. Tarnec, S. Muth, E. Montagnon, J. Poree, and G. Cloutier, "Stolt's f-k migration for plane wave ultrasound imaging," IEEE Transactions on Ultrasonics, Ferroelectrics, and Frequency Control, vol. 60, no. 9, pp. 1853-1867, 2013.

[12] C. Fan, M. Pan, F. Luo, and B. Drinkwater, "Multi-frequency time-reversal-based imaging for ultrasonic nondestructive evaluation using full matrix capture," IEEE Transactions on Ultrasonics, Ferroelectrics, and Frequency Control, vol. 61, no. 12, pp. 2067-2074, 2014.

[13] C. Holmes, B. W. Drinkwater, and P. D. Wilcox, "Postprocessing of the full matrix of ultrasonic transmit-receive array data for non-destructive evaluation," NDT \& E International, vol. 38, no. 8, pp. 701-711, 2005.

[14] M. Sutcliffe, M. Weston, B. Dutton, P. Charlton, and K. Donne, "Real-time full matrix capture for ultrasonic nondestructive testing with acceleration of post-processing through graphic hardware," NDT \& E International, vol. 51, pp. 16-23, 2012.

[15] M. Njiki, A. Elouardi, S. Bouaziz, O. Casula, and O. Roy, “A multi-FPGA architecture-based real-time TFM ultrasound imaging," Journal of Real-Time Image Processing, vol. 16, no. 2, pp. 505-521, 2019.

[16] A. J. Hunter, B. W. Drinkwater, and P. D. Wilcox, "The wavenumber algorithm for full-matrix imaging using an ultrasonic array," IEEE Transactions on Ultrasonics, Ferroelectrics, and Frequency Control, vol. 55, no. 11, pp. 2450-2462, 2008. 
[17] H. Hu, J. Du, C. Ye, and X. Li, "Ultrasonic phased array sparse-TFM imaging based on sparse array optimization and new edge-directed interpolation," Sensors, vol. 18, no. 6, p. 1830, 2018.

[18] X. Zhao, T. Z. Qi, and Z. Y. Wang, "Ultrasonic detection triangle matrix focus imaging algorithm," Journal of $\mathrm{Me}$ chanical Engineering, vol. 55, no. 4, pp. 19-24, 2019.

[19] X. F. Guo, Y. Han, and P. F. Nie, "Ultrasound imaging algorithm: half-matrix focusing method based on reciprocity," Mathematical Problems in Engineering, vol. 2021, Article ID 8888469, 11 pages, 2021.

[20] M. H. Skjelvareid and Y. Birkelund, "Ultrasound imaging using multilayer synthetic aperture focusing," in Proceedings of the ASME Pressure Vessels and Piping Division/K-PVP Conference American Society of Mechanical Engineers Digital Collection, pp. 1-9, Bellevue, WA, USA, July 2010.

[21] T. Olofsson, "Phase shift migration for imaging layered objects and objects immersed in water," IEEE Transactions on Ultrasonics, Ferroelectrics, and Frequency Control, vol. 57, no. 11, pp. 2522-2530, 2010.

[22] C. H. Chih-Hsiung Chang, Y. F. Young-Fo Chang, Y. Yushieh Ma, and K. K. Shung, "Reliable estimation of virtual source position for SAFT imaging," IEEE Transactions on Ultrasonics, Ferroelectrics, and Frequency Control, vol. 60, no. 2, pp. 356-363, 2013.

[23] J. F. Cruza and J. Camacho, "Total focusing method with virtual sources in the presence of unknown geometry interfaces," IEEE Transactions on Ultrasonics, Ferroelectrics, and Frequency Control, vol. 63, no. 10, pp. 1581-1592, 2016.

[24] M. H. Skjelvareid, T. Olofsson, Y. Birkelund, and Y. Larsen, "Synthetic aperture focusing of ultrasonic data from multilayered media using an omega-K algorithm," IEEE Transactions on Ultrasonics, Ferroelectrics, and Frequency Control, vol. 58, no. 5, pp. 1037-1048, 2011.

[25] R. H. Stolt, "Migration by fourier transform," Geophysics, vol. 43, no. 1, pp. 23-48, 1978.

[26] H. Wu, J. Chen, K. Yang, and X. Hu, "Ultrasonic array imaging of multilayer structures using full matrix capture and extended phase shift migration," Measurement Science and Technology, vol. 27, no. 4, Article ID 045401, 2016.

[27] T. Lukomski, "Full-matrix capture with phased shift migration for flaw detection in layered objects with complex geometry," Ultrasonics, vol. 70, pp. 241-247, 2016.

[28] H. Jin and J. Chen, "An efficient wavenumber algorithm towards real-time ultrasonic full-matrix imaging of multilayered medium," Mechanical Systems and Signal Processing, vol. 149, Article ID 107149, 2021.

[29] H. W. Hu, J. Du, Y. Li, and Z. G. Zhou, "Two-layer medium ultrasonic phased array total focusing method imaging based on sparse matrix," Journal of Mechanical Engineering, vol. 53, no. 14, pp. 128-135, 2017.

[30] W. A. Schneider, “The common depth point stack," Proceedings of the IEEE, vol. 72, no. 10, pp. 1238-1254, 1984.

[31] P. S. Schultz, "Seismic velocity estimation," Proceedings of the IEEE, vol. 72, no. 10, pp. 1330-1339, 1984.

[32] A. Rointan, M. Soleimani Monfared, and H. Aghajani, "Improvement of seismic velocity model by selective removal of irrelevant velocity variations," Acta Geodaetica et Geophysica, vol. 56, no. 1, pp. 145-176, 2021.

[33] R. Anvari, A. R. Kahoo, M. S. Monfared, M. Mohammadi, R. M. D. Omer, and A. H. Mohammed, "Random noise attenuation in seismic data using Hankel sparse low-rank approximation," Computers \& Geosciences, vol. 153, Article ID 104802, 2021. 